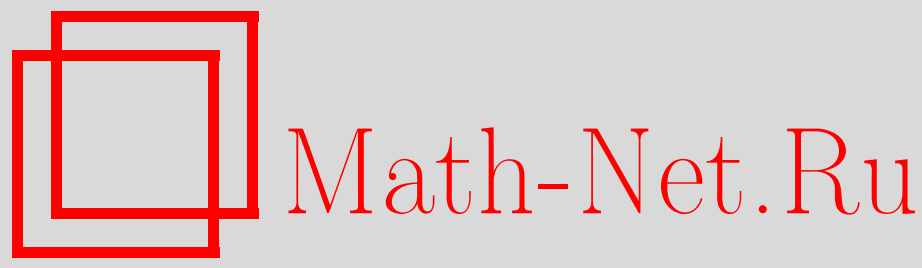

Е. И. Зеленов, Об эквивалентности коммутационных соотношений, ТМФ, 2008, том 157, номер 3, 406-412

DOI: https://doi.org/10.4213/tmf6288

Использование Общероссийского математического портала Math-Net.Ru подразумевает, что вы прочитали и согласны с пользовательским соглашением http://www . mathnet.ru/rus/agreement

Параметры загрузки:

IP : 54.162 .27 .143

26 апреля 2023 г., 13:03:10

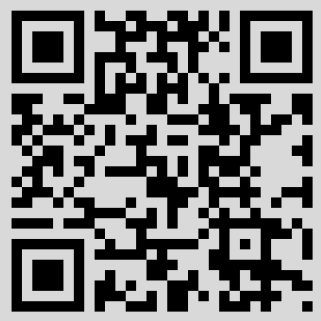




\section{ОБ ЭКВИВАЛЕНТНОСТИ КОММУТАЦИОННЫХ СООТНОШЕНИЙ}

Рассматриваются $\mathcal{C}^{*}$-алгебры коммутационных соотношений над полями $\mathbb{Q}_{p}$, $p=2,3,5, \ldots, \infty$. Описаны все неприводимые сепарабельные представления вышеуказанных алгебр. Доказано, что алгебры не изоморфны при различных $p$.

Ключевые слова: коммутационные соотношения, p-адическая топология, неприводимые представления, эквивалентность представлений.

\section{1. ОСНОВНЫЕ ОПРЕДЕЛЕНИЯ}

Пусть $\mathbb{Q}, \mathbb{R}, \mathbb{C}$ - поля рациональных, вещественных и комплексных чисел соответственно. Через $\mathbb{Q}_{p}, p=2,3,5, \ldots$, обозначим поля $p$-адических чисел, $\mathbb{Q}_{\infty}=\mathbb{R}$ (т.е. “ $\infty$-адический" всюду в дальнейшем будет означать "вещественный”). Аддитивная группа поля обозначается таким же символом, что и поле. Все поля снабжены стандартной топологией. Если будет рассматриваться другая топология, отличная от стандартной, то это будет особо отмечаться.

Пусть $G$ - локально-компактная абелева группа, $\widehat{G}$ - группа характеров группы $G$ (двойственная по Понтрягину). Группу $G$ будем записывать аддитивно, группу $\widehat{G}-$ мультипликативно.

ОПРЕДЕЛЕНИЕ 1. Представлением коммутационных соотношений над группой $G$ назовем пару $(\mathcal{W}, \mathcal{H})$, где $\mathcal{H}$ - гильбертово пространство над $\mathbb{C}, \mathcal{W}$ - отображение из $G \times \widehat{G}$ в множество унитарных операторов в $\mathcal{B}(\mathcal{H})(\mathcal{B}(\mathcal{H})$ - алгебра ограниченных операторов на $\mathcal{H})$, удовлетворяющее следующим условиям:

1) $\mathcal{W}(0,1)=\operatorname{Id}_{\mathcal{H}}$;

2) отображение $(g, \hat{g}) \mapsto \mathcal{W}(g, \hat{g})$ непрерывно в слабой операторной топологии;

3) $\mathcal{W}(g, \hat{g}) \mathcal{W}(h, \hat{h})=\hat{g}(h) \overline{\hat{h}(g)} \mathcal{W}(h, \hat{h}) \mathcal{W}(g, \hat{g})$.

Понятия цикличности, неприводимости, унитарной эквивалентности и прямой суммы представлений коммутационных соотношений определяются естественным образом. Известно (теорема единственности Стоуна-фон Неймана-Макки) [1], [2],

*E-mail: evgeny.zelenov@gmail.com 
что существует единственное с точностью до унитарной эквивалентности неприводимое представление коммутационных соотношений, а любое представление коммутационных соотношений есть прямая сумма неприводимых представлений (это доказано для более общего случая произвольной локально-компактной группы).

В дальнейшем мы также будем рассматривать разрывные представления коммутационных соотношений (т.е. представления, для которых свойство непрерывности 2 из определения 1 не выполняется).

Докажем простое следствие определения коммутационных соотношений, которое показывает, что любое представление является точным.

Лемма 1. Пусть $(\mathcal{W}, \mathcal{H})$ - представление коммутационных соотношений над группой $G$. Справедливо следующее утверждение. Если $\mathcal{W}(g, \hat{g})=\operatorname{Id}_{\mathcal{H}}$, то $g=0$, $\hat{g}=1$, m.е. $(g, \hat{g})$ - единичный элемент группы $G \times \widehat{G}$.

ДоказАтельство. Пусть $(g, \hat{g}) \in G \times \widehat{G}, g \neq 0, \hat{g} \neq 1, \mathcal{W}(g, \hat{g})=\operatorname{Id}_{\mathcal{H}}$. Найдется такой элемент $\hat{h} \in \widehat{G}$, что $\hat{h}(g) \neq 1$ (т.е. множество характеров разделяет точки в $G$ ). Поскольку $\mathcal{W}(g, \hat{g})=\operatorname{Id}_{\mathcal{H}}$, равенство $\langle W(g, \hat{g}) \phi, \phi\rangle_{\mathcal{H}}=1$ верно для любого элемента $\phi \in \mathcal{H},\|\phi\|=1$. Таким образом,

$$
\begin{aligned}
1 & =\langle\mathcal{W}(g, \hat{g}) \phi, \phi\rangle_{\mathcal{H}}=\langle\mathcal{W}(0, \hat{h}) \mathcal{W}(g, \hat{g}) \phi, \mathcal{W}(0, \hat{h}) \phi\rangle_{\mathcal{H}}= \\
& =\hat{h}(g)\langle\mathcal{W}(g, \hat{g}) \mathcal{W}(0, \hat{h}) \phi, \mathcal{W}(0, \hat{h}) \phi\rangle_{\mathcal{H}}=\hat{h}(g) \neq 1
\end{aligned}
$$

Полученное противоречие показывает, что $g=0$. Аналогичными рассуждениями доказывается, что $\hat{g}=1$.

Заметим, что при доказательстве мы не использовали непрерывность операторов $\mathcal{W}(g, \hat{g})$.

Из теоремы единственности следует корректность следующего определения.

ОПРЕДЕЛЕНИЕ 2. Пусть $(\mathcal{W}, \mathcal{H})$ - неприводимое представление коммутационных соотношений. $\mathcal{C}^{*}$-алгеброй $\mathcal{A}(G)$ коммутационных соотношений над $G$ назовем $\mathcal{C}^{*}$-подалгебру $\mathcal{B}(H)$, порожденную одномерным проектором и семейством операторов $\{\mathcal{W}(g, \hat{g}), g \in G, \hat{g} \in \widehat{G}\}$.

В силу теоремы Стоуна-фон Неймана-Макки алгебра коммутационных соотношений $\mathcal{A}(G)$ над $G$ не зависит от выбора представления (различные представления определяют изоморфные алгебры). Представление $(\mathcal{W}, \mathcal{H})$ из определения 2 будем называть представлением коммутационных соотношений, порождающим алгебру $\mathcal{A}_{p}$. Заметим, что условие неприводимости коммутационных соотношений в определении алгебры можно не учитывать.

\section{2. АЛГЕБРА $\mathcal{A}_{p}=\mathcal{A}\left(\mathbb{Q}_{p}\right)$}

Для случая $\mathbb{Q}_{p}, p=2,3,5, \ldots, \infty$, группы $\mathbb{Q}_{p}$ и $\widehat{\mathbb{Q}}_{p}$ изомофны. Изоморфизм задается формулой $\mathbb{Q}_{p} \ni y \rightarrow \hat{y}(x)=\chi_{p}(y x) \in \widehat{\mathbb{Q}}_{p}, x \in \mathbb{Q}_{p}$, где $\chi_{p}-$ произвольный (фиксированный) невырожденный характер группы $\mathbb{Q}_{p}$. В качестве $\chi_{p}$ можно выбрать характер $\chi_{p}(x)=e^{2 \pi i\{x\}_{p}}$, где $\{x\}_{p}-p$-адическая дробная часть $x$. В случае 
$G=\mathbb{Q}_{p}$ коммутационные соотношения имеют вид

$$
\mathcal{W}(x, y) \mathcal{W}\left(x^{\prime}, y^{\prime}\right)=\chi_{p}\left(x y^{\prime}-y x^{\prime}\right) \mathcal{W}\left(x^{\prime}, y^{\prime}\right) \mathcal{W}(x, y)
$$

Удобно ввести следующее обозначение. Пусть $z=(x, y), z^{\prime}=\left(x^{\prime}, y^{\prime}\right)$. Обозначим через $B$ симплектическую форму на $\mathbb{Q}_{p} \times \mathbb{Q}_{p}: B\left(z, z^{\prime}\right)=x y^{\prime}-y x^{\prime}$.

Пусть непрерывные коммутационные соотношения $(\mathcal{W}, \mathcal{H})$ порождают алгебру $\mathcal{A}_{p}$. Представление $\left(\pi_{0}, \mathcal{H}\right)$ алгебры $\mathcal{A}_{p}$ в алгебре $\mathcal{B}(\mathcal{H})$ ограниченных операторов на $\mathcal{H}$, задаваемое соотношением $\pi_{0}(\mathcal{W}(z))=\mathcal{W}(z)$, будем называть тождественным представлением алгебры $\mathcal{A}_{p}$.

Условие непрерывности коммутационных соотношений приводит к следующему существенному свойству алгебры $\mathcal{A}_{p}$.

Лемма 2. Пусть $(\mathcal{W}, \mathcal{H})$ - произвольное непрерьвное представление коммутаиионных соотношений (не обязательно неприводимое), порождающее алгебру $\mathcal{A}_{p}$. Тогда алгебра фон Неймана, порожденная тождественным представлением $\mathcal{A}_{p}$ в $\mathcal{B}(\mathcal{H})$, сепарабельна.

ДокАЗАтельство. Пусть $(\mathcal{W}, \mathcal{H})$ - представление коммутационных соотношений, порождающее алгебру $\mathcal{A}_{p}$. Поскольку отображение

$$
z \mapsto \mathcal{W}(z), \quad z \in \mathbb{Q}_{p} \times \mathbb{Q}_{p},
$$

слабо непрерывно, то счетное семейство операторов $\{\mathcal{W}(q), q \in \mathbb{Q} \times \mathbb{Q}\}$ образует плотное подмножество в алгебре фон Неймана, порожденной операторами $\{\mathcal{W}(z), z \in$ $\left.\mathbb{Q}_{p} \times \mathbb{Q}_{p}\right\}$. Следовательно, указанная выше алгебра фон Неймана счетно порождена и тем самым сепарабельна.

Важным классом представлений алгебры $\mathcal{A}_{p}$ являются представления, содержащие одномерный проектор. Они имеют ясный физический смысл. А именно, это фоковские представления, а указанный выше проектор - это проектор на вакуумное состояние. Неприводимые представления, содержащие одномерный проектор, содержат алгебру компактных операторов $\mathcal{K}(\mathcal{H})$ [3].

Тождественное представление $\pi_{0}$ - единственное представление, содержащее компактные операторы. А именно, справедлива следующая лемма.

Лемма 3. Пусть $(\mathcal{W}, \mathcal{H})$ - непрерывное неприводимое представление, порождающее алгебру $\mathcal{A}_{p},\left(\pi, \mathcal{H}_{\pi}\right)$ - произвольное неприводимое представление алгебрь $\mathcal{A}_{p}$. Тогда либо $\pi$ эквивалентно $\pi_{0}$, либо $\pi(\mathcal{K}(\mathcal{H}))=0$.

ДоказАтельство. Действительно, пусть $K \in \mathcal{K}(\mathcal{H})$ и $\pi(K) \neq 0$. Тогда $\pi\left(\mathcal{A}_{p}\right)$ содержит компактный оператор. В силу неприводимости представления $\pi$ образ $\pi\left(\mathcal{A}_{p}\right)$ содержит алгебру компактных операторов $\mathcal{K}\left(\mathcal{H}_{\pi}\right) \subseteq \pi\left(\mathcal{A}_{p}\right)$ [3]. Поскольку алгебра компактных операторов имеет единственное с точностью до эквивалентности неприводимое представление, то

$$
\left.\left.\pi\right|_{\mathcal{K}\left(\mathcal{H}_{\pi}\right)} \simeq \pi_{0}\right|_{\mathcal{K}(\mathcal{H})}
$$

В силу единственности продолжения представления $\pi$ с алгебры $\mathcal{K}\left(\mathcal{H}_{\pi}\right)$ на $\pi\left(\mathcal{A}_{p}\right)$ получаем требуемое утверждение. 
Из леммы 3 вытекает следующая теорема.

Теорема 1. Алгебры $\mathcal{A}_{p}$ и $\mathcal{A}_{p^{\prime}}$ изоморфнъ тогда и толъко тогда, когда $p=p^{\prime}$.

ДоказАтЕЛьство. Пусть $(\mathcal{W}, \mathcal{H}),\left(\mathcal{W}^{\prime}, \mathcal{H}^{\prime}\right)$ - неприводимые представления коммутационных соотношений, порождающие алгебры $\mathcal{A}_{p}$ и $\mathcal{A}_{p^{\prime}}$ соответственно, и пусть $\pi: \mathcal{A}_{p} \rightarrow \mathcal{A}_{p^{\prime}}$ - изоморфизм. Поскольку $\pi(\mathcal{W}(z)), z \in \mathbb{Q}_{p} \times \mathbb{Q}_{p}$, удовлетворяют коммутационным соотношениям алгебры $\mathcal{A}_{p}$, то $\left(\pi, \mathcal{H}^{\prime}\right)$ - неприводимое представление алгебры $\mathcal{A}_{p}$ в $\mathcal{B}\left(\mathcal{H}^{\prime}\right)$, при этом $\operatorname{Ker}(\pi)=0$. Следовательно (см. лемму 3$)$, $\pi$ эквивалентно тождественному представлению и $\mathcal{W}(z)$ и $\mathcal{W}^{\prime}(z)$ должны удовлетворять одним и тем же коммутационным соотношениям. Значит, для любого рационального числа $q$ должно выполняться равенство $\chi_{p}(q)=\chi_{p^{\prime}}(q)$, что возможно только при $p=p^{\prime}$.

Несложно построить пример разрывного представления коммутационных соотношений. Действительно, пусть $\lambda: \mathbb{Q}_{p} \times \mathbb{Q}_{p} \rightarrow \mathbb{C}$ - разрывный характер аддитивной группы $\mathbb{Q}_{p} \times \mathbb{Q}_{p}$, а $(\mathcal{W}, \mathcal{H})$ - непрерывное представление коммутационных соотношений. Рассмотрим представление коммутационных соотношений $\left(\mathcal{W}_{\lambda}, \mathcal{H}\right)$, где $\mathcal{W}_{\lambda}(z)=\lambda(z) \mathcal{W}(z), z \in \mathbb{Q}_{p} \times \mathbb{Q}_{p}$. Легко видеть, что операторы из семейства $\left\{\mathcal{W}_{\lambda}(z)\right.$, $\left.z \in \mathbb{Q}_{p} \times \mathbb{Q}_{p}\right\}$ удовлетворяют коммутационным соотношениям, но при этом отображение $z \mapsto \mathcal{W}_{\lambda}(z)$ не является непрерывным в слабой топологии. Кроме того, поскольку представление $\left(\mathcal{W}_{\lambda}, \mathcal{H}\right)$ разрывно, оно не является унитарно-эквивалентным исходному представлению $(\mathcal{W}, \mathcal{H})$.

Справедлива следующая лемма.

Лемма 4. Пусть $\lambda$ - характер (возможно, разрывный) группь $\mathbb{Q}_{p} \times \mathbb{Q}_{p}$. Представление коммутационных соотношений $\left(\mathcal{W}_{\lambda}, \mathcal{H}\right)$ неприводимо тогда и только тогда, когда неприводимо исходное представление $(\mathcal{W}, \mathcal{H})$.

ДокАЗАтЕЛьСтво. Пусть $A \in \mathcal{B}(\mathcal{H})$ - произвольный ограниченный оператор на $\mathcal{H}$. Справедлива формула

$$
\left[A, \mathcal{W}_{\lambda}(z)\right]=\lambda(z)[A, \mathcal{W}(z)], \quad z \in \mathbb{Q}_{p} \times \mathbb{Q}_{p}
$$

Поскольку $\lambda(z)$ не равно нулю ни при каких значениях $z \in \mathbb{Q}_{p} \times \mathbb{Q}_{p}$, оператор $A$ коммутирует со всеми операторами $\mathcal{W}_{\lambda}(z)$ тогда и только тогда, когда он коммутирует со всеми операторами $\mathcal{W}(z)$. Дальнейшее следует из определения неприводимости коммутационных соотношений.

Лемма 5. Пусть $(\mathcal{W}, \mathcal{H})$ - непрерывное представление коммутационных соотношений, $\lambda, \tilde{\lambda}$ - характерь (возможсно, разрьвные) группы $\mathbb{Q}_{p} \times \mathbb{Q}_{p}$. Представления коммутационных соотношений $\left(\mathcal{W}_{\lambda}, \mathcal{H}\right),\left(\mathcal{W}_{\tilde{\lambda}}, \mathcal{H}\right)$ унитарно-эквивалентны тогда и только тогда, когда $\lambda^{-1} \tilde{\lambda}-$ непрерьвный характер группь $\mathbb{Q}_{p} \times \mathbb{Q}_{p}$.

ДокАзАтЕльство. Для доказательства леммы достаточно показать, что если $\lambda$ - непрерывный характер группы $\mathbb{Q}_{p} \times \mathbb{Q}_{p}$, то представления $(\mathcal{W}, \mathcal{H})$ и $\left(\mathcal{W}_{\lambda}, \mathcal{H}\right)$ унитарно-эквивалентны. Поскольку $\lambda$ - непрерывный характер, то существует 
$\left(a, a^{\prime}\right) \in \mathbb{Q}_{p} \times \mathbb{Q}_{p}$ такое, что для всех $z=\left(x, x^{\prime}\right) \in \mathbb{Q}_{p} \times \mathbb{Q}_{p}$ справедливо равенство $\lambda\left(x, x^{\prime}\right)=\chi_{p}\left(-x a^{\prime}+x^{\prime} a\right)[4]$. При этом, как легко заметить из определения коммутационных соотношений,

$$
\mathcal{W}_{\lambda}\left(x, x^{\prime}\right)=\mathcal{W}\left(a, a^{\prime}\right) \mathcal{W}\left(x, x^{\prime}\right) \mathcal{W}^{-1}\left(a, a^{\prime}\right)
$$

для всех $z=\left(x, x^{\prime}\right) \in \mathbb{Q}_{p} \times \mathbb{Q}_{p}$.

Разрывные характеры группы $\mathbb{Q}_{p} \times \mathbb{Q}_{p}$ удобно рассматривать как непрерывные характеры той же группы, но с дискретной топологией.

Пусть $\mathbb{Q}_{p}^{d}$ - аддитивная группа поля $\mathbb{Q}_{p}$ с дискретной топологией. Справедлива следующая лемма.

Лемма 6. Группа $\mathbb{Q}_{p}^{d}$ изоморфна прямой сумме $\bigoplus_{\alpha \in \Omega} \mathbb{Q}$ континуума экземпляров аддитивной группы поля рациональных чисел $\mathbb{Q}$ (множество $\Omega$ - континуум, $|\Omega|=c)$.

Доказательство для случая аддитивной группы поля вещественных чисел с дискретной топологией (в обозначениях настоящей работы $-\mathbb{Q}_{\infty}^{d}$ ) можно найти в работе [4]. Для произвольного $p$ доказательство аналогично.

Удобно ввести следующее обозначение. Пусть $\mathbb{D}_{p}=\mathbb{Q}_{p}^{d} \times \mathbb{Q}_{p}^{d}, \widehat{\mathbb{D}}_{p}$ - соответствующая группа характеров. Согласно лемме $6 \mathbb{D}_{p}$ изоморфны при разных $p$, в дальнейшем индекс $p$ будем опускать. Заметим, что $\widehat{\mathbb{D}}$ компактна и изоморфна прямому произведению континуума экземпляров группы характеров поля рациональных чисел $\left(\widehat{\mathbb{D}}\right.$ - компактификация Бора локально-компактной группы $\left.\mathbb{Q}_{p} \times \mathbb{Q}_{p}\right)$. Непрерывные (в обычной топологии на $\mathbb{Q}_{p}$ ) характеры группы $\mathbb{Q}_{p} \times \mathbb{Q}_{p}$ образуют плотную подгруппу в $\widehat{\mathbb{D}}$, фактор-группа $\widehat{\mathbb{D}} / \mathbb{Q}_{p} \times \mathbb{Q}_{p}$ имеет мощность $2^{c}$ [4].

Пространство $\mathbb{D}$ имеет следующую физическую интерпретацию. Одной из идей, лежащих в основе $p$-адической математической физики [5], является утверждение о том, что результатом физического измерения является рациональное (а не вещественное) число. В продолжение этой идеи можно утверждать, что результатом физического эксперимента, т.е. конечной серией измерений, является конечная последовательность рациональных чисел, а именно, элемент пространства $\mathbb{D}$ (см. лемму 6).

Пусть $(\mathcal{W}, \mathcal{H})$ - неприводимое представление коммутационных соотношений, порождающее алгебру $\mathcal{A}_{p}$. Отображение $\mathcal{A}_{p} \rightarrow \mathcal{A}_{p}: \mathcal{W}(x) \mapsto \lambda(x) \mathcal{W}(x), x \in \mathbb{Q}_{p} \times \mathbb{Q}_{p}$, где $\lambda \in \widehat{\mathbb{D}}$, задает гомоморфизм алгебры $\mathcal{A}_{p}$ в себя. Таким образом, определено представление $\left(\pi_{\lambda}, \mathcal{H}\right)$ алгебры $\mathcal{A}_{p}$. Свойства построенных представлений перечислены в следующей теореме.

Теорема 2. Пусть $(\mathcal{W}, \mathcal{H})$ - неприводимое представление коммутационных соотношений, порождающее алгебру $\mathcal{A}_{p}, \lambda \in \widehat{\mathbb{D}}-$ характер группь $\mathbb{D},\left(\pi_{\lambda}, \mathcal{H}\right)$ - представление алгебры $\mathcal{A}_{p}$, задаваемое на образующих алгебры соотношением $\pi_{\lambda}(\mathcal{W}(z))$ $=\lambda(z) \mathcal{W}(z), z \in \mathbb{Q}_{p} \times \mathbb{Q}_{p}$. Представление $\left(\pi_{\lambda}, \mathcal{H}\right)$ обладает следующими свойствами:

1) $\left(\pi_{\lambda}, \mathcal{H}\right)$ неприводимо; 
2) $\pi_{\lambda} \simeq \pi_{\lambda^{\prime}}$ тогда и только тогда, когда $\lambda^{-1} \lambda^{\prime}-$ непрерывный характер группь $\mathbb{Q}_{p} \times \mathbb{Q}_{p}$

3) $\pi_{\lambda} \simeq \pi_{0}$ тогда и только тогда, когда $\lambda$ - непрерывный характер группы $\mathbb{Q}_{p} \times \mathbb{Q}_{p}$

4) если $\lambda$ - разрывныц характер, то $\pi_{\lambda}(K)=0$ для любого $K \in \mathcal{K}(\mathcal{H})$.

ДокАзАтЕльство. Утверждение 1 следует из леммы 4; утверждение 2 следует из леммы 5; утверждение 3 является прямым следствием утверждения 2; утверждение 4 вытекает из леммы 3 и утверждений 1 и 3 . Теорема доказана.

Заметим, что алгебра $\mathcal{A}_{p}$ не является простой, так как в противном случае она совпадала бы с алгеброй компактных операторов и имела бы единственное с точностью до эквивалентности неприводимое представление. Остается выяснить, является ли алгебра $\mathcal{A}_{p} / \mathcal{K}(\mathcal{H})$ простой. Изучение алгебры $\mathcal{A}_{p} / \mathcal{K}(\mathcal{H})$ представляет значительный интерес, поскольку именно эта алгебра описывает различия алгебр $\mathcal{A}_{p}$ при различных $p$. Некоторые свойства алгебры $\mathcal{A}_{p} / \mathcal{K}(\mathcal{H})$ отражены в следующей теореме.

Теорема 3. Пусть $(\mathcal{W}, \mathcal{H})$ - неприводимое представление коммутационных соотношений, порождающее алгебру $\mathcal{A}_{p}$. Множество классов унитарно-эквивалентных неприводимых представлений алгебры $\mathcal{A}_{p} / \mathcal{K}(\mathcal{H})$ в сепарабелъном гилъбертовом пространстве находится во взаимно однозначном соответствии с элементами фактор-группъ $\widehat{\mathbb{D}} / \mathbb{D}$.

ДоказАтЕЛьство. Пусть $\left(\mathcal{W}^{\prime}, \mathcal{H}^{\prime}\right)$ - неприводимое представление коммутационных соотношений (не обязательно непрерывное). Можно показать [6], что существует такой характер $\lambda$ группы $\mathbb{D}$, что представление $\left(\mathcal{W}_{\lambda}^{\prime}, \mathcal{H}^{\prime}\right)$ слабо измеримо. Слабо измеримое представление локально-компактной группы в сепарабельном гильбертовом пространстве слабо непрерывно. Заметим, что для несепарабельного пространства это неверно [4]. Таким образом, всякое неприводимое представление алгебры $\mathcal{A}_{p}$ в сепарабельном гильбертовом пространстве имеет вид $\pi_{\lambda}$. Дальнейшие свойства следуют из теоремы 2.

При каждом $p$ отображение $\mathbb{D}_{p} \rightarrow \widehat{\mathbb{D}}$ задает однопараметрическую подгруппу в $\widehat{\mathbb{D}}[4]$, причем $p$-адическая топология определяет способ вложения $\mathbb{D}_{p}$ в $\widehat{\mathbb{D}}$. Таким образом, теорема 3 задает соответствие между однопараметрическими подгруппами в $\widehat{\mathbb{D}}$ и неприводимыми представлениями, содержащими вакуумный вектор. Вышеуказанные однопараметрические подгруппы можно интерпретировать как "время". В этих терминах p-адическому “времени" соответствует непрерывное в $p$-адической топологии представление коммутационных соотношений, содержащее вакуумный вектор.

Несложно построить пример циклического слабо измеримого представления алгебры $\mathcal{A}_{p} / \mathcal{K}(\mathcal{H})$, которое не является непрерывным (см. пример Тома в [4]). В качестве пространства представления выберем несепарабельное пространство $\mathcal{L}_{2}(\mathbb{D})$, 
циклический вектор $\phi_{0}(0)=1$ и $\phi_{0}(z)=0$ в остальных точках. Искомое представление на образующих $\mathcal{W}(z), z \in \mathbb{Q}_{p} \times \mathbb{Q}_{p}$, алгебры $\mathcal{A}_{p}$ зададим соотношением

$$
[\pi(\mathcal{W}(z)) \phi](s)=\chi_{p}(B(z, s)) \phi(s-z) .
$$

Построенное представление слабо измеримо относительно меры Хаара на $\mathbb{Q}_{p} \times \mathbb{Q}_{p}$, поскольку для любых $\phi, \psi \in \mathcal{L}_{2}(\mathbb{D})$ функция $z \mapsto(\mathcal{W}(z) \phi, \psi)$ отлична от нуля не более чем в счетном числе точек, при этом она не является непрерывной. Осталось показать, что построенное представление является представлением алгебры $\mathcal{A}_{p} / \mathcal{K}(\mathcal{H})$. Действительно, это представление является Гельфанда-Наймарка-Сигала представлением для состояния $\rho$ на алгебре $\mathcal{A}_{p}$, где $\rho$ на образующих алгебры определяется соотношением $\rho(\mathcal{W}(z))=1$, если $z=0$, и равно нулю в других точках. Легко убедиться [6], что $\rho$ является состоянием типа следа на $\mathcal{A}_{p}$, следовательно $\rho(\mathcal{K}(\mathcal{H}))=0$, поскольку на алгебре компактных операторов нет ненулевых состояний типа следа. Следовательно, построенное представление определяет представление алгебры $\mathcal{A}_{p} / \mathcal{K}(\mathcal{H})$.

Благодарности. Автор выражает благодарность сотрудникам отдела математической физики Математического института им. В. А. Стеклова РАН за плодотворные обсуждения и внимание к его работе. Работа выполнена в рамках Программы поддержки ведущих научных школ (грант НШ-3224.2008.1).

\section{Список литературы}

[1] G. W. Mackey, Duke Math. J., 16 (1949), 311-326.

[2] M. Rieffel, Duke Math. J., 39 (1972), 745-752.

[3] Дж. Мёрфи, $C^{*}$-алгебры и теория операторов, Факториал, М., 1997.

[4] Э. Хьюитт, К. Росс, Абстрактный гармонический анализ, Т. 1, 2, Наука, М., 1975.

[5] В. С. Владимиров, И. В. Волович, Е. И. Зеленов, р-Адический анализ и математическая физика, Наука, М., 1994.

[6] J. Slawny, Comm. Math. Phys., 24:2 (1972), 151-170.

Поступила в редакцию 7.04.2008 